\title{
Les neutrinos n'ont pas livré tous leurs secrets
}

Stéphane Lavignac ${ }^{(1)}$ (stephane.lavignac@cea.fr) et Marco Zito ${ }^{(2)}$ (marco.zito@cea.fr)

(1) Institut de physique théorique, Orme des Merisiers, CEA-Saclay, 91191 Gif-sur-Yvette Cedex

(2) Service de physique des particules, DSM/IRFU, CEA-Saclay, 91191 Gif-sur-Yvette Cedex

Le prix Nobel de physique

2015 récompense plusieurs

décennies de recherches

ayant abouti à la découverte

des oscillations des neutrinos,

propriété que ces particules

ont de se transformer

les unes dans les autres

au cours de leur mouvement.

Cette particularité implique

qu'ils ont une masse, dont

l'origine, à ce jour inconnue,

pourrait dépasser le cadre

du Modèle Standard, la théorie

de référence des particules

élémentaires.

De nombreuses autres

questions restent par ailleurs en suspens : quelle est l'échelle de masse des neutrinos?

Les antineutrinos oscillent-ils comme les neutrinos?

Le neutrino est-il sa propre

antiparticule?

Un vaste programme

expérimental vise à répondre

à ces questions, et à quelques autres.
Le neutrino est une particule élémentaire de $\operatorname{spin} 1 / 2$ (un fermion), dépourvue de charge électrique. Insensible aux interactions électromagnétique et forte, il n'entre pas dans la composition des atomes, contrairement à l'électron et aux quarks, mais est produit dans de nombreuses réactions gouvernées par l'interaction faible. Son existence fut postulée par le physicien autrichien Wolfgang Pauli en 1930. Elle expliquait le spectre continu des électrons émis par la radioactivité bêta, qui semblait violer le principe de la conservation de l'énergie. Alors que dans les autres transitions nucléaires, l'énergie de la particule émise (photon ou particule alpha) prend une valeur bien déterminée, correspondant à la différence $Q$ entre l'énergie de masse de l'état initial et celle de l'état final, l'énergie des électrons bêta peut prendre n'importe quelle valeur entre zéro et $Q$. Pauli suggéra donc que dans la désintégration bêta, l'électron est accompagné par une autre particule - légère et n'interagissant quasiment pas avec la matière - avec laquelle il partage l'énergie émise dans la réaction : le neutrino. Ce dernier était alors considéré comme de masse nulle, ce qui était cohérent avec toutes les observations expérimentales avant la découverte des oscillations.

La détection des neutrinos restera impossible pendant plusieurs décennies. Deux avancées majeures permettront de les faire passer d'une hypothèse à une réalité observée et mesurable.

La première est due à Enrico Fermi qui proposa, en 1934, la première théorie de la désintégration bêta, basée sur une nouvelle force fondamentale, l'interaction faible. Grâce à cette théorie, les physiciens purent calculer le taux d'interaction des neutrinos avec la matière. Ce dernier est extraordinairement faible, inférieur, pour un neutrino d'un gigaélectron-volt $\left(1 \mathrm{GeV}=10^{9} \mathrm{eV}\right)$, de quinze ordres de grandeur à celui d'un photon de même énergie.

La deuxième avancée est d'ordre expérimental. Depuis 1942, les physiciens savent construire des réacteurs nucléaires. Dans le cœur de ceux-ci, les noyaux issus de la fission de l'uranium et du plutonium, très riches en neutrons, subissent une série de désintégrations bêta, produisant d'énormes quantités d'antineutrinos (environ $510^{20}$ par seconde pour une centrale d'un gigawatt). C'est donc auprès d'un réacteur qu'aura lieu la première expérience qui détectera des neutrinos (en réalité, comme on le saura plus tard, des antineutrinos électroniques $\bar{v}_{\mathrm{e}}$ ), sur le site de Savannah River aux USA, en 1956. Cette découverte vaudra le prix Nobel de physique à Frederick Reines en 1995. Ayant ainsi établi l'existence de cette particule, les physiciens se tourneront tout naturellement vers l'étude d'autres sources intenses de neutrinos, à commencer par le Soleil. Non sans avoir auparavant découvert une deuxième espèce de neutrino, le neutrino muonique $v_{\mu}$, en 1962 (prix Nobel 1988), puis, en 2000 seulement, une troisième espèce de neutrino, le neutrino tauique $v_{\tau}$.

L'observation des neutrinos solaires et atmosphériques (issus des rayons cosmiques) mettra bientôt les physiciens sur la piste des oscillations (encadré 1), dont l'étude se poursuit aujourd'hui dans le cadre de collaborations internationales, au sein desquelles les équipes françaises jouent un rôle actif. La découverte de ces oscillations a des conséquences profondes (voir l'encadré 2, p. 6). 

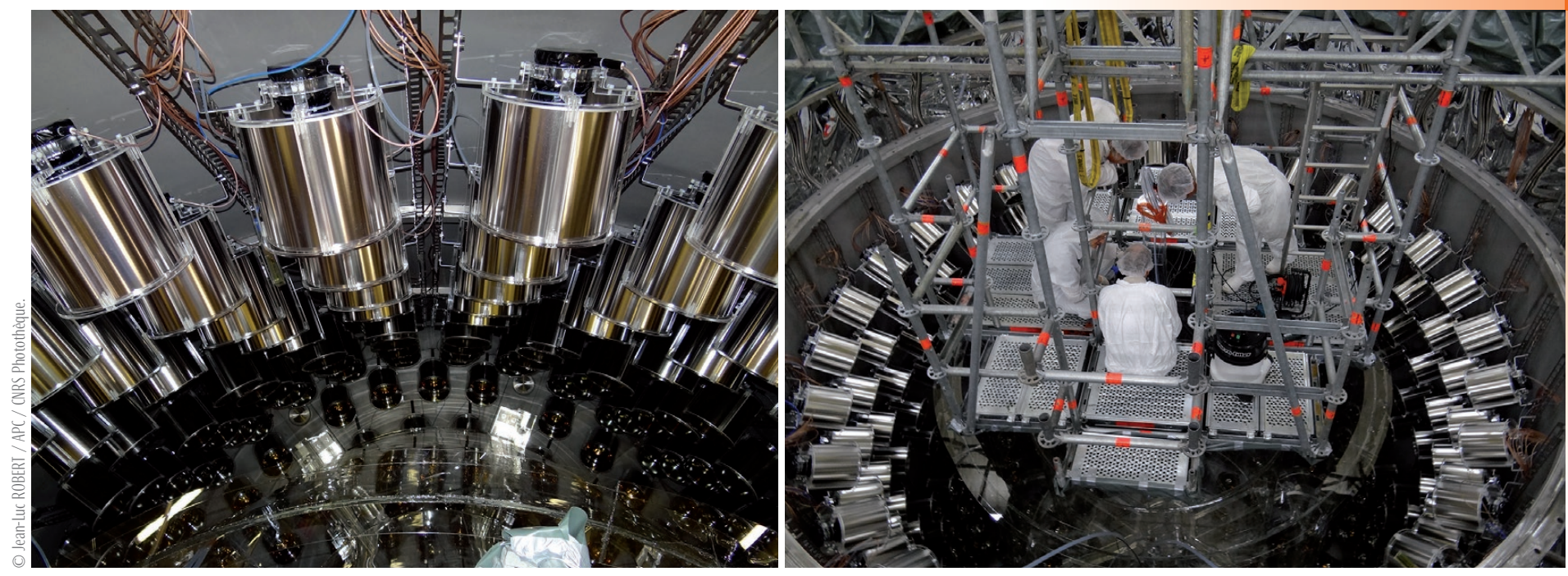

Photomultiplicateurs tapissant les parois de la cuve du second détecteur de l'expérience Double Chooz, sur le site de la centrale nucléaire de Chooz dans les Ardennes. Cette expérience a pour but de mesurer le troisième angle de mélange des neutrinos, $\theta_{13}$ (voir pp. 7-8).

\section{Les oscillations de neutrinos}

Le neutrino électronique $\left(v_{\mathrm{e}}\right)$ découvert en 1956 est le partenaire, pour l'interaction faible à l'origine de la désintégration bêta, de l'électron. Mais ce dernier possède des cousins aux propriétés identiques, à l'exception de leur masse : le muon, environ 200 fois plus lourd que l'électron, et le tau, près de 3500 fois plus lourd. Leurs partenaires respectifs sont les neutrinos muonique $\left(v_{u}\right)$ et tauique $\left(v_{\tau}\right)$. On appelle saveur leptonique le nombre quantique qui les distingue; ainsi, $v_{\mathrm{e}}, v_{\mu}$ et $v_{\tau}$ sont des états propres de saveur.

Supposons à présent que les neutrinos aient une masse ; en général, les états propres de saveur ne coïncident pas avec les états propres de masse $v_{1}, v_{2}$ et $v_{3}$, mais sont des superpositions cohérentes de ces derniers. Dans un cadre simplifié à deux neutrinos, on a par exemple $\left|v_{\mathrm{e}}\right\rangle=\cos \theta\left|v_{1}\right\rangle+\sin \theta\left|v_{2}\right\rangle$, où $\theta$ est appelé angle de mélange. Les états propres de masse étant également des états propres du Hamiltonien (du moins en l'absence d'interactions, c'est-à-dire dans le vide), la mécanique quantique nous enseigne que chacun d'eux acquiert une phase proportionnelle à son énergie au cours du temps, de sorte qu'un neutrino produit initialement dans l'état $\mid v_{\mathrm{e}}>$ sera décrit par la combinaison $\cos \theta \mathrm{e}^{-\mathrm{i} \mathrm{E}_{1} t / \hbar}\left|v_{1}>+\sin \theta \mathrm{e}^{-\mathrm{i} \mathrm{E}_{2} t / \hbar}\right| v_{2}>$ après avoir parcouru une distance $\mathrm{L} \cong \mathrm{ct}$. Autrement dit, l'état physique du neutrino change au cours de son mouvement. Il est donc possible de le détecter dans une saveur différente de celle dans laquelle il a été produit : $c^{\prime}$ est le phénomène d'oscillation. La probabilité qu'un neutrino de saveur $\alpha$ ait oscillé en une autre saveur $\beta$ après avoir parcouru une distance $L$ est donnée par $P\left(v_{\alpha} \rightarrow v_{\beta}\right)=\sin ^{2} 2 \theta \sin ^{2}\left(\Delta \mathrm{m}^{2} \mathrm{~L} / 4 \mathrm{E}\right)$, où $\Delta \mathrm{m}^{2}=\mathrm{m}_{2}^{2}-\mathrm{m}_{1}^{2}$ est la différence des carrés des masses des états propres de masse et $\mathrm{E}$ est l'énergie du neutrino. L'amplitude des oscillations est donc déterminée par l'angle de mélange, tandis que la longueur d'oscillation est proportionnelle au rapport E/ $\Delta \mathrm{m}^{2}$ (fig. E1). On notera que les oscillations ne dépendent pas directement des masses des neutrinos, mais seulement de la différence de leurs carrés.

Par ailleurs, les oscillations des neutrinos sont un exemple remarquable de conservation des propriétés de cohérence quantique sur des distances macroscopiques, puisqu'elles ont été observées pour des neutrinos ayant traversé la Terre (voir « La découverte des oscillations des neutrinos atmosphériques », p. 6).

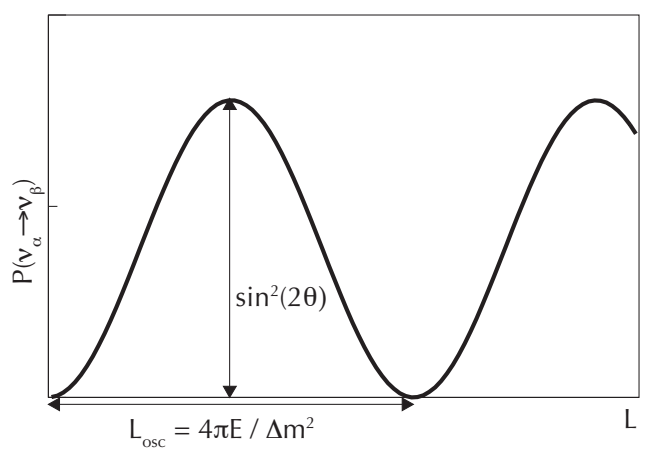

E1. Probabilité d'oscillation en fonction de la distance L parcourue par le neutrino.

\section{Les déficits des neutrinos solaires et atmosphériques}

Les neutrinos solaires sont des neutrinos électroniques produits dans les réactions thermonucléaires à l'origine de l'énergie du Soleil, la majeure partie d'entre eux provenant de la fusion de deux protons en deutérium $\left(\mathrm{p}+\mathrm{p} \rightarrow \mathrm{D}+\mathrm{e}^{+}+\mathrm{v}_{\mathrm{e}}\right)$. Leur spectre, qui s'étend de $0,1 \mathrm{MeV}$ à $20 \mathrm{MeV}$ environ, est prédit par les modèles solaires. L'expérience menée par Raymond Davis (prix Nobel 2002) dans la mine de Homestake, fut la première, dès la fin des années 1960, à détecter les neutrinos solaires. Cette expérience, qui utilisait pour cela la réaction $v_{\mathrm{e}}+{ }^{37} \mathrm{Cl} \rightarrow{ }^{37} \mathrm{Ar}+\mathrm{e}^{-}$, observa un tiers seulement des neutrinos électroniques prédits. Au cours des années 1990, d'autres expériences confirmèrent ce qui fut bientôt connu sous le nom de déficit ou problème des neutrinos solaires. GALLEX (en Italie) et SAGE (dans le Caucase russe), expériences souterraines utilisant la transition gallium-germanium $v_{\mathrm{e}}+{ }^{71} \mathrm{Ga} \rightarrow{ }^{71} \mathrm{Ge}+\mathrm{e}^{-}$, observèrent un déficit sur une plus grande partie du spectre des neutrinos solaires, grâce à un seuil de détection plus bas que l'expérience de Davis. Au Japon, Kamiokande et SuperKamiokande utilisèrent une autre méthode de mesure, consistant à détecter la lumière Tcherenkov émise dans un grand volume d'eau par les électrons accélérés par leurs collisions avec des neutrinos solaires de haute énergie (diffusion élastique $v+\mathrm{e}^{-} \rightarrow v+\mathrm{e}^{-}$). Le déficit des neutrinos solaires était désormais bien établi, mais la preuve expérimentale qu'il était dû à des oscillations, comme le théoricien Bruno Pontecorvo l'avait suggéré dès 1968, manquait toujours. Il fallut attendre pour 
$>>$

cela les résultats décisifs de l'expérience SNO (Sudbury Neutrino Observatory) au Canada, en 2001-2002.

Entretemps, une autre anomalie était apparue lors de l'étude des neutrinos produits par les rayons cosmiques, ces particules de haute énergie en provenance du cosmos, constituées en majorité de protons. Leurs interactions avec les noyaux atomiques de l'atmosphère produisent des gerbes de particules, notamment des pions $\pi^{ \pm}$, qui se désintègrent selon les réactions $\pi^{ \pm} \rightarrow \mu^{ \pm}+v_{\mu}$ suivies de $\mu^{ \pm} \rightarrow \mathrm{e}^{ \pm}+v_{\mathrm{e}}+v_{\mu}$. Ces neutrinos, dits atmosphériques, dont l'énergie s'étend de quelques centaines de $\mathrm{MeV}$ jusqu'à des dizaines de $\mathrm{GeV}$ ou plus, peuvent traverser la Terre de part en part. À partir des années 1960, les premières expériences souterraines commencent à les détecter, et certaines d'entre elles mesurent un flux de neutrinos muoniques inférieur aux prédictions.

Les expériences SNO et Super-Kamiokande allaient résoudre ces deux énigmes en prouvant qu'elles sont dues au phénomène d'oscillation, c'est-à-dire à la propriété qu'ont les neutrinos de différentes saveurs de se transformer les uns dans les autres au cours de leur mouvement (voir encadré 1).

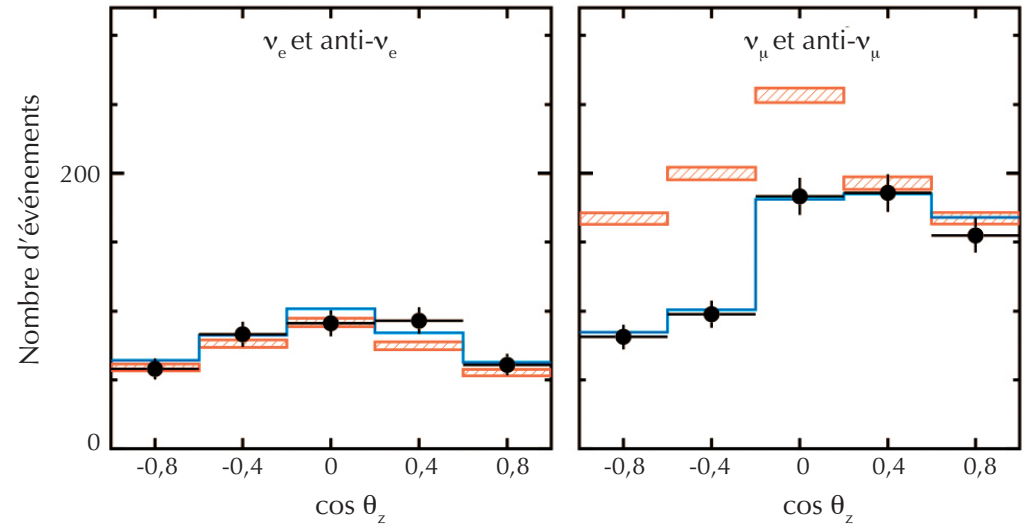

1. Distribution, en fonction de l'angle zénithal, des événements de neutrinos atmosphériques (de type $v_{e}$ à gauche, de type $v_{u}$ à droite) d'énergie supérieure à 1,33 GeV observés par Super-Kamiokande (d’après [1]). Les histogrammes orange représentent les prédictions en l'absence d'oscillations, les bleus représentent les prédictions avec oscillations, tandis que les points noirs correspondent aux données.

\section{La découverte des oscillations des neutrinos atmosphériques}

C'est grâce à une nouvelle expérience, Super-Kamiokande, qu'on put véritablement établir l'existence des oscillations des neutrinos atmosphériques. Son détecteur est situé dans la mine de Kamioka, dans les Alpes japonaises. La roche de la montagne, d'une épaisseur de mille mètres, fait écran aux rayons cosmiques et permet de détecter des processus rares comme les interactions des neutrinos.

\section{Une découverte aux conséquences profondes}

Le fait que les neutrinos oscillent, nous l'avons vu dans l'encadré 1, implique qu'ils ont une masse. Cette observation en apparence anodine est en fait révolutionnaire, le Modèle Standard de la physique des particules postulant que les neutrinos sont de masse nulle.

En quoi les masses des neutrinos seraient-elles différentes de celles des autres particules? Tout d'abord, elles sont extrêmement faibles, environ $10^{6}$ fois plus petites que celle de l'électron. Ensuite, étant électriquement neutre, le neutrino a la possibilité d'être sa propre antiparticule : on parle de fermion de Majorana, les autres constituants élémentaires de la matière étant des fermions de Dirac. Supposer que les neutrinos sont des fermions de Majorana permet d'expliquer la petitesse de leurs masses, et rend possibles de nouveaux phénomènes associés à la violation du nombre leptonique, dont l'origine pourrait se trouver dans une théorie plus fondamentale que le Modèle Standard, valable à haute énergie. Défini comme le nombre quantique égal à +1 pour un neutrino ou un lepton chargé négativement comme l'électron, et à -1 pour leurs antiparticules, le nombre leptonique est accidentellement conservé dans le Modèle Standard, mais violé en présence de neutrinos de Majorana (auxquels on ne peut associer de nombre leptonique, puisqu'ils sont à la fois neutrino et antineutrino). Parmi les conséquences de sa violation figure, outre la double désintégration bêta sans émission de neutrino [2], la possibilité d'expliquer l'origine de l'asymétrie matière-antimatière de l'Univers par le mécanisme de la leptogenèse (voir l'encadré 3, p. 8).
Ce détecteur est constitué d'une grande cuve de 50000 tonnes d'eau ultra-pure, observée par près de 11000 photomultiplicateurs de grande dimension. L'interaction d'un neutrino avec l'eau de la cuve peut produire des particules chargées ultrarelativistes, notamment des électrons ou des muons. Ceux-ci se déplacent à une vitesse supérieure à celle de la lumière dans l'eau et y produisent de la lumière Tcherenkov, sous la forme d'un cône de lumière autour de leurs trajectoires. Les caractéristiques de ces photons Tcherenkov permettent, entre autres, de mesurer la direction de la particule chargée, d'en estimer l'énergie si elle s'arrête dans le détecteur, et de déterminer s'il s'agit d'un muon ou d'un électron.

Super-Kamiokande permet d'étudier en détail les neutrinos atmosphériques grâce à son grand volume et aux bonnes performances du détecteur.

C'est à la conférence Neutrino 1998 que les résultats d'une analyse établissant l'existence des oscillations des neutrinos atmosphériques furent présentés (fig. 1). Si l'on considère la distribution des événements de type muon en fonction de l'angle zénithal $\theta_{\mathrm{z}}$ des neutrinos incidents, on observe un déficit très marqué par rapport à la prédiction pour les neutrinos montants $\left(\cos \theta_{\mathrm{z}}<0\right)$, mais pas pour les neutrinos descendants $\left(\cos \theta_{z}>0\right)$. Cela s'explique par le fait que la probabilité d'oscillation dépend de la distance. Les neutrinos descendants n'ont parcouru qu'une dizaine de kilomètres avant d'interagir dans le détecteur, tandis que les neutrinos montants ont parcouru 


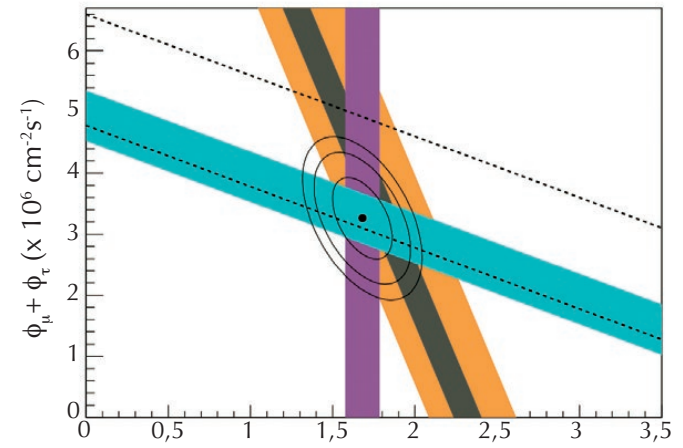

$\phi_{\mathrm{e}}\left(\mathrm{x} 10^{6} \mathrm{~cm}^{-2} \mathrm{~s}^{-1}\right)$

2. Flux de neutrinos muoniques et tauiques $\left(\phi_{\mu}+\phi_{\tau}\right)$ versus flux de neutrinos électroniques $\left(\phi_{\mathrm{e}}\right)$ provenant du Soleil (d'après B. Aharmim et al., Phys. Rev. C 72 (2005) 055502). Les bandes violette, orange et turquoise correspondent à des combinaisons distinctes de ces flux mesurées par SNO via différentes réactions, avec leurs incertitudes expérimentales à 1o. La bande marron est la mesure de Super-Kamiokande. L'intersection de ces bandes détermine les valeurs observées de $\phi_{e}$ et $\phi_{u}+\phi_{\tau}$, indiquées par le point noir et des ellipses correspondant à différents niveaux de confiance statistique (68\%, 95\% et 99\%). Enfin, la région entre les deux lignes en pointillés est la prédiction du modèle solaire de Bahcall, Serenelli et Basu (2005), qui doit être comparée au flux total $\phi_{\mathrm{e}}{ }^{+} \phi_{\mu}+\phi_{\tau}$ mesuré par SNO (bande turquoise).

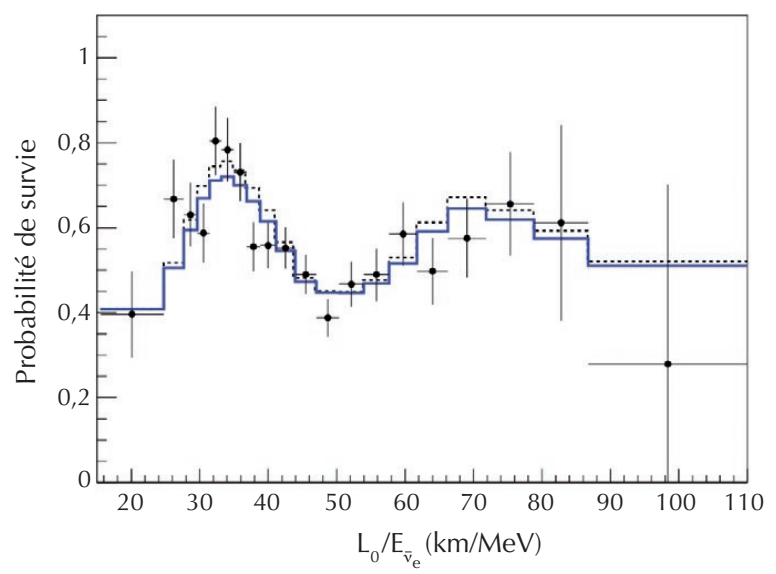

3. Oscillations de neutrinos issus de réacteurs nucléaires. Fraction d’antineutrinos électroniques observés par l'expérience KamLAND (par rapport au flux attendu en l'absence d'oscillations) en fonction du rapport $\mathrm{L}_{0} / \mathrm{E}$, où $L_{0}$ est la distance moyenne parcourue par les $\bar{v}_{\mathrm{e}}$ et $\mathrm{E}$ leur énergie (d'après A. Gando et al., Phys. Rev. D 83 (2011) 052002). Les données et les incertitudes expérimentales sont figurées par les points noirs et les barres horizontales et verticales, tandis que l'histogramme bleu correspond aux valeurs attendues en présence d'oscillations. Le deuxième maximum d'oscillation (creux vers $\mathrm{L}_{0} / \mathrm{E}=50 \mathrm{~km} / \mathrm{MeV}$ ) apparaît clairement dans les données.

jusqu'à $12700 \mathrm{~km}$ (le diamètre terrestre). L'interprétation de ces résultats est que les neutrinos muoniques ont oscillé en neutrinos de type tau avec un angle de mélange proche de la valeur maximale $\pi / 4$, et une longueur d'oscillation de l'ordre du diamètre de la Terre.

\section{La découverte des oscillations des neutrinos solaires}

Le grand apport de SNO, détecteur à effet Tcherenkov utilisant de l'eau lourde $\left(\mathrm{D}_{2} \mathrm{O}\right)$ pour cible, fut de mesurer à la fois le flux total $\phi_{\mathrm{e}}+\phi_{\mu}+\phi_{\tau}$ des neutrinos provenant du Soleil (a), grâce à la réaction

\section{Oscillations avec des sources artificielles de neutrinos}

À la suite de SNO et de SuperKamiokande, une série d'expériences s'est attachée à confirmer les oscillations observées avec des sources artificielles de neutrinos (antineutrinos électroniques issus de réacteurs nucléaires, et faisceaux de neutrinos ou d'antineutrinos muoniques produits par des accélérateurs) et à mesurer précisément les paramètres dont elles dépendent.

Les expériences K2K (au Japon) et MINOS (aux USA) ont confirmé les oscillations observées par Super-Kamiokande avec des faisceaux produits par un accélérateur, dont les caractéristiques sont mieux connues que celles des neutrinos atmosphériques. Ces faisceaux, résultant de la désintégration des pions produits par la collision de protons incidents sur une cible, parcourent ensuite plusieurs centaines de kilomètres dans la croûte terrestre avant d'être détectés. L'expérience OPERA a mis en évidence les oscillations $v_{\mu} \rightarrow v_{\tau}$ en observant la production de neutrinos de type tau dans un faisceau de neutrinos muoniques produit au CERN et dirigé vers le laboratoire souterrain du Gran Sasso, en Italie.

Les résultats de SNO ont été confirmés dès 2003 par l'expérience japonaise KamLAND, qui a observé la disparition d'une fraction des antineutrinos électroniques produits par les centrales nucléaires environnantes. Interprétées en termes d'oscillations, les données de KamLAND, de $\mathrm{SNO}$ et des autres expériences " solaires » s'expliquent par des valeurs communes des paramètres $\Delta \mathrm{m}^{2}$ et $\sin ^{2} 2 \theta$. Par ailleurs, l'origine oscillatoire de la disparition des $\bar{v}_{\mathrm{e}}$ est clairement visible dans les résultats de KamLAND (fig. 3).

$v+\mathrm{D} \rightarrow \boldsymbol{v}+\mathrm{p}+\mathrm{n}$ (à laquelle les trois saveurs de neutrinos contribuent de la même façon), et le flux des neutrinos électroniques $\phi_{\mathrm{e}}$, via la réaction $v_{\mathrm{e}}+\mathrm{D} \rightarrow \mathrm{e}^{-}+\mathrm{p}+\mathrm{p}$. Le flux total mesuré par SNO est en accord avec la prédiction des modèles solaires, alors que le flux des neutrinos électroniques est nettement inférieur, indiquant de façon claire une transformation de ces derniers en neutrinos d'autres saveurs, comme on s'y attend en présence d'oscillations $^{(b)}$. Ces résultats décisifs, illustrés par la figure 2, ont valu le prix Nobel de physique 2015 à Arthur B. McDonald, porte-parole de la collaboration SNO depuis 1990.
Plus récemment, un nouveau canal d'oscillation associé à un troisième angle de mélange (appelé $\theta_{13}$ ) a été mis en évidence. Les premières indications ont été données à l'été 2011 par l'expérience japonaise T2K [3], dans laquelle un faisceau de neutrinos muoniques produits par un accélérateur de protons est dirigé vers le détecteur de Super-Kamiokande, 295 km plus loin. Ces résultats ont été confirmés par une nouvelle génération d'expériences auprès de réacteurs nucléaires (dont Double Chooz en France et Daya Bay en Chine), la première mesure de précision ayant été fournie par Daya Bay. T2K a, par ailleurs, 


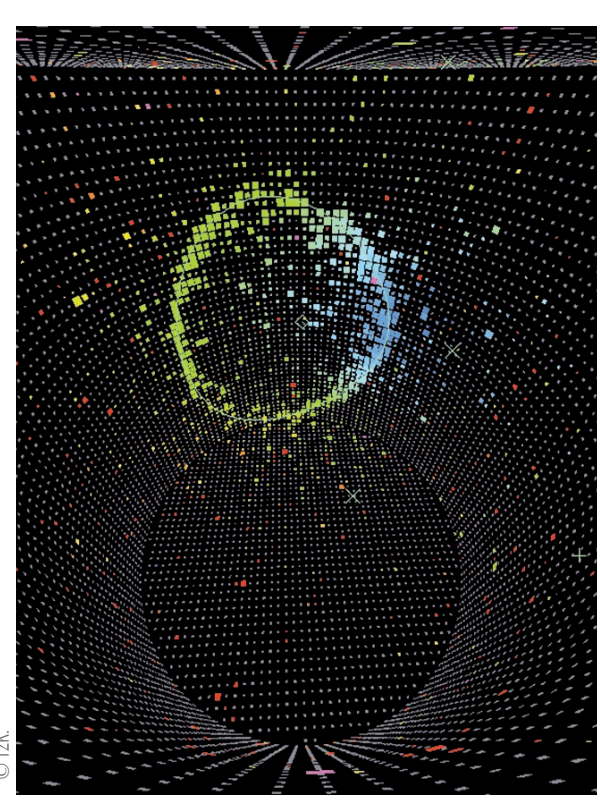

4. Événement observé par Super-Kamiokande, résultant de l'interaction d'un neutrino électronique du faisceau de l'expérience $\mathrm{T} 2 \mathrm{~K}$ avec l'eau de la cuve. Chaque point de couleur correspond à la détection de lumière Tcherenkov par un des photomultiplicateurs localisés sur les parois de la cuve, la couleur étant fonction de la quantité de lumière détectée. L'anneau de forme circulaire correspond à la position reconstruite du cône de lumière Tcherenkov.

\section{Masses des neutrinos}

encadré 3

\section{et asymétrie matière-antimatière de l'Univers}

L'un des plus grands mystères de la physique et de la cosmologie contemporaine est l'origine de l'asymétrie matière-antimatière, c'est-à-dire du fait que l'Univers est essentiellement composé de baryons (protons et neutrons) et d'électrons, sans présence significative d'antimatière.

En 1967, le physicien soviétique Andreï Sakharov énonça les conditions pour la création dynamique d'une asymétrie baryonique ou baryogenèse. Parmi celles-ci figurent l'existence de processus qui ne conservent pas le nombre de baryons, ainsi que la violation de la symétrie $\mathrm{CP}^{(\mathrm{c})}$, afin que les taux de production des baryons et des antibaryons soient différents. Bien que ces ingrédients soient présents dans le Modèle Standard, ce dernier échoue à engendrer l'asymétrie observée. Dans les années 1980, les physiciens japonais M. Fukugita et T. Yanagida ont proposé un mécanisme alternatif, la leptogenèse. Ce scénario fait intervenir des particules très massives, par ailleurs impliquées dans la génération des masses des neutrinos. Produites dans l'univers primordial, elles se désintègrent avec des taux différents en leptons et antileptons. Cette asymétrie leptonique est ensuite convertie en asymétrie baryonique par des processus non perturbatifs du Modèle Standard. S'il n'est guère envisageable de tester directement la leptogenèse, il est possible de vérifier expérimentalement si les conditions nécessaires sont satisfaites : la violation du nombre leptonique, intimement liée à la nature de fermion de Majorana des neutrinos, par l'observation de la double désintégration bêta sans émission de neutrino ; et la violation de la symétrie $\mathrm{CP}$, par la mise en évidence de probabilités différentes pour les oscillations de neutrinos et d'antineutrinos.

C'est à cette dernière tâche que travaillent actuellement les expériences T2K au Japon et NOvA aux États-Unis, avec deux projets d'envergure prévus pour la prochaine décennie, Hyper-Kamiokande et DUNE, qui utiliseront des faisceaux de neutrinos intenses et de grands détecteurs souterrains. Ces derniers constitueront également des observatoires de neutrinos de supernovae et permettront de poursuivre la recherche de la désintégration du proton, qui est prévue par les théories de Grande Unification, dont certaines prédisent par ailleurs l'existence des états lourds nécessaires à la leptogenèse. propriétés d'une interaction de neutrino) ou scintillateurs liquides. Ces nouveaux projets permettront de déterminer le spectre de masse des neutrinos (c'est-à-dire l'ordonnancement de leurs masses, seules les différences de leurs carrés étant connues) et de rechercher une éventuelle violation de la symétrie CP dans les oscillations (voir l'encadré 3). Pour le premier point, des approches alternatives existent, fondées sur l'étude de précision des oscillations des antineutrinos émis par des centrales nucléaires, ou sur celles des neutrinos atmosphériques.

D'autres questions fondamentales sont abordées par des expériences ne faisant pas appel aux oscillations. L'échelle absolue de la masse des neutrinos pourrait être déterminée par les observations cosmologiques (la limite supérieure actuelle sur la somme des masses, due au satellite Planck, est $0,23 \mathrm{eV}$ ) ou par l'étude du spectre des électrons dans la désintégration bêta. La nature du neutrino, fermion de Dirac ou de Majorana, est explorée par la recherche de la double désintégration bêta sans émission de neutrino $(Z, A) \rightarrow(Z+2, A)+2 \mathrm{e}^{-}$. Ce processus viole le nombre leptonique et n'est possible que pour des neutrinos de Majorana.

Enfin, certaines anomalies expérimentales restant à confirmer suggèrent l'existence d'un nouveau type de neutrino, des neutrinos stériles, ainsi dénommés parce qu'ils ne sont pas sensibles à l'interaction faible (ils ne seraient donc sensibles qu'à la gravité). Cette hypothèse sera testée prochainement par des expériences auprès de réacteurs nucléaires.

Objet d'étude, le neutrino est également une excellente sonde astrophysique. Son très faible taux d'interaction avec la matière en fait un messager de choix pour nous renseigner sur des phénomènes très lointains ou qui se produisent dans des milieux très denses. Ainsi, l'observation des neutrinos solaires a permis de confirmer notre compréhension de ce qui se passe au centre du Soleil. L'observation des neutrinos de la supernova 1987A a inauguré l'astronomie des neutrinos. Récemment, l'expérience IceCube (télescope à neutrinos enfoui dans la glace de l'Antarctique) a observé des neutrinos d'énergie supérieure au $\mathrm{PeV}\left(10^{15} \mathrm{eV}\right)$, dont l'origine n'a pas encore été identifiée. L'Europe développe de son côté un projet de télescope à neutrinos, KM3NeT (voir la brève, p. 9). 


\section{Conclusion}

La découverte des oscillations des neutrinos a couronné des années d'intenses recherches expérimentales et permis de démontrer que ces particules ont une masse, ouvrant une fenêtre sur d'hypothétiques nouvelles lois physiques, qui pourraient être à l'origine de l'asymétrie matièreantimatière de l'Univers. Cependant, en dépit de ces succès, les neutrinos n'ont pas encore livré tous leurs secrets. L'origine de leur masse reste inconnue, ainsi que leur nature (sont-ils des fermions de Dirac ou de Majorana ?). Nous ne savons toujours pas si la symétrie CP est violée dans le secteur des leptons, ni s'il existe d'autres espèces de neutrinos. De nombreuses expériences, en cours ou projetées, visent à répondre à ces questions. Elle promettent une belle moisson de résultats dans les années à venir, et, espérons-le, des surprises de taille, comme celles qui jalonnent l'histoire des neutrinos.

(a) Il s'agit en réalité, comme pour l'expérience Super-Kamiokande, de la partie de haute énergie du spectre des neutrinos solaires (la réaction de dissociation du deutérium $v+\mathrm{D} \rightarrow \mathrm{v}+\mathrm{p}+\mathrm{n}$ ayant un seuil en énergie de $E_{v}=2,225 \mathrm{MeV}$ ), c'est-à-dire des neutrinos produits dans le centre du Soleil par la réaction ${ }^{8} \mathrm{~B} \rightarrow{ }^{8} \mathrm{Be}+\mathrm{e}^{+}+v_{\mathrm{e}}$ (" neutrinos du bore $\left."\right)$.

(b) Une description précise des transitions $v_{\mathrm{e}} \rightarrow v_{\mu} / \nu_{\tau}$ à l'intérieur du Soleil nécessite la prise en compte des interactions entre les neutrinos et les électrons, protons et neutrons du milieu. Ces « effets de matière " ont été étudiés par les théoriciens Lincoln Wolfenstein en 1978, et Stanislas Mikheïev et Alekseï Smirnov en 1985-1986.

(c) Une transformation de charge-parité consiste à inverser les coordonnées d'espace d'un système physique (transformation de parité) et à remplacer toutes les particules par leurs antiparticules. Dans le Modèle Standard électrofaible, la symétrie CP n'est violée que par une phase présente dans le secteur des quarks, constituants du proton et du neutron.

\section{Références}

1• "Neutrino oscillations" (Scientific background on the Nobel Prize in Physics 2015, en anglais), www.nobelprize.org/nobel_prizes/physics/ laureates/2015/advanced-physicsprize2015.pdf .

2• C. Marquet et L. Simard, « Le neutrino est-il identique à son antiparticule ?",

Reflets de la Physique 24 (2011) 11-14.

3- F. Vannucci, «Quelques faits saillants de la conférence Neutrino 2012 », Reflets de la Physique 31 (2012) 20-21.

4 J. Bouchez, "La saga des neutrinos ",

Bulletin de la Société Française de Physique 132 (2001) 9-14. 